\title{
The Double Loop Sigma Delta Modulator with Unstable Filter Dynamics: Stability Analysis and Tone Behavior
}

\author{
Mariam Motamed, Seth Sanders, and Avideh Zakhor
}

\begin{abstract}
Conventional $\Sigma \Delta$ modulators suffer from idle tones. In order to alleviate the tone problem, $\Sigma \Delta$ modulators with unstable filter dynamics have been proposed. In this paper we present an analysis of the saturation characteristics and tone behavior of the double loop $\Sigma \Delta$ modulator with unstable filter dynamics. We begin by deriving stability boundaries for the double loop $\Sigma \Delta$ modulator with unstable filter dynamics which yield bounds on maximum internal signal levels. We then show via simulations and steady state analysis that while tonal properties are improved by using unstable filter dynamics, idle tones are not completely removed. Specifically, we show that some unstable limit cycles have an attractor region in their neighborhood which resultsin tones in the spectrum corresponding to the fundamental or harmonics of these limit cycles.
\end{abstract}

\section{INTRODUCTION}

$\mathbf{O}$ VERSAMPLED $\Sigma \Delta$ modulators are becoming increasingly important in data conversion applications. Their advantage over conventional conversion methods lies in their robustness to circuit imperfections and component nonidealities. The principle behind their operation is to tradeoff sampling rate with amplitude resolution. Specifically, $\Sigma \Delta$ modulators sample signals many times faster than the Nyquist rate, but only with one bit of amplitude resolution. Low-pass filtering is typically used to recover the signal from the high speed stream of one bit amplitude quantized output.

An important factor affecting the performance of $\Sigma \Delta$ modulators is their tone behavior. Tones are spectral peaks in the signal baseband that especially limit the use of $\Sigma \Delta$ modulators in demanding audio applications, such as in compact disc players. It has been shown that single loop and double loop modulators, and possibly other classes of encoders, exhibit nonwhite, discrete quantization noise spectra for dc inputs [1], [2]. In the case of single loop modulators, the location and relative power of these tones have been derived analytically [1], while for double loop modulators, the evidence is mostly empirical [2]. Even though the tone problem is generally considered to be less severe for higher order modulators, they are not immune from basic tone problems associated with lower order ones [3].

The traditional tone removal technique consists of using additive dither signals to randomize the bit pattern at the output

Manuscript received December 18, 1994; revised November 1, 1995. This paper was recommended by Associate Editor D. A. Johns.

The authors are with the Department of Electrical Engineering and Computer Science, University of California, Berkeley, CA 94720 USA.

Publisher Item Identifier S 1057-7130(96)04571-5. of $\Sigma \Delta$ modulators [3]. A more recent approach to alleviate the tone problem consists of using unstable filters in $\Sigma \Delta$ modulators. This approach stems from relating the discrete tones in the quantization noise spectra of $\Sigma \Delta$ modulators to their limit cycle behavior. Consequently, the tone mitigation problem may be treated as that of preventing the internal states of the modulator from locking into periodic orbits. Specifically, for the first-order modulator, Feely [4] has shown that while an integrator pole inside the unit circle results in asymptotically stable limit cycles, moving the pole outside the unit circle results in unstable limit cycles ${ }^{1}$.

Recently, there has been a number of new results on improving the tone behavior by using $\Sigma \Delta$ structures with unstable filter dynamics ${ }^{2}$ [5]-[11]. In [5] we present simulation results showing that the tone properties of higher order $\Sigma \Delta$ modulators are influenced by the location of the poles of the open loop transfer function. In [6], we discuss the tradeoff between tones, saturation and Signal-to-Noise Ratio (SNR) of the double loop modulator with respect to pole location, and obtain saturation bounds on the integrator states. Schreier [7] discusses the tradeoff between SNR and tonality of firstand second-order modulators as a function of pole location. Hein [8] obtains an approximate measure of tone persistence in double loop $\Sigma \Delta$ modulators as a function of pole location, and extends the bounding technique of [12] to obtain bounds on state variables. In our previous paper [9], we investigate the tonal behavior of the double loop $\Sigma \Delta$ modulator with unstable filter dynamics. Risbo [10] suggests introducing an all-pass term with a zero near -1 in the noise transfer function prototype of $\Sigma \Delta$ modulators in order to alleviate high frequency tones. The stability of this method is achieved by increasing the modulator's order. Reference [11] shows simulation results comparing the performance of $\Sigma \Delta$ modulators with open loop poles outside the unit circle to that of dithered modulators.

In this paper, we present an analysis of double loop $\Sigma \Delta$ modulators with unstable filter dynamics with the specific goal of determining their saturation characteristics and tonal properties. The first part of this paper is concerned with stability of the internal states of the double loop modulator.

\footnotetext{
'Asymptotically stable limit cycles are periodic orbits about which all sufficiently small perturbations tend to zero. Note that, only asymptotically stable limit cycles persist and are observed in practice. Unstable limit cycles, on the other hand, do not persist since, infinitesimal perturbations of the state space trajectory result in divergence from the periodic orbit.

${ }^{2}$ Note that, $\Sigma \Delta$ modulators with unstable filter dynamics are modulators with one or more open loop poles outside the unit circle.
} 
Specifically, we obtain two continuous time approximations to the double loop modulator whose asymptotic behaviors are solved numerically, and show that the asymptotic behavior of the continuous time systems provides stability boundaries for the internal states of the discrete time modulator. We then investigate the tonal properties of the double loop modulator. We find that while moving the open loop poles outside the unit circle improves tonal content, it does not completely remove idle tones. We identify a mechanism causing spectral tones, outline a numerical method to study this mechanism, and illustrate our points by considering a zero dc input example. In all that follows, our analysis and simulations assume constant encoder input. This assumption follows from the fact that the input to the modulator is oversampled and therefore may be approximated by a constant. In addition, it has been shown that the tone behavior is worse when the input to the modulator is constant or slowly varying.

The paper is organized as follows. In Section II, we present a brief overview of the problem considered in this paper. In Sections III and IV we discuss the saturation properties of the double loop $\Sigma \Delta$ modulator with unstable filter dynamics, and obtain bounds on the internal signal levels. In Section V, we discuss the effect of pole location on the maximum allowable input dynamic range and SNR performance. In Section VI we analyze the tone behavior in the modulator with unstable filter dynamics by analyzing the steady state behavior about unstable limit cycles. Finally, in Section VII we present a summary of our results. All simulations are done with MATLAB.

\section{LIMIT CyClE STABILITY}

Consider the double loop $\Sigma \Delta$ modulator shown in Fig. 1. The modulator consists of two integrators, and a 1-b quantizer $Q$, embedded in a negative feedback loop. Signals $u(n)$ and $v(n)$ denote the states, $D$ denotes a unit delay element, and $p_{1}$ and $p_{2}$ are the open loop poles. The quantizer is specified by $Q(u)=+1$ for $u>0$, and $Q(u)=-1$ for $u \leq 0$. The constant input $X$ is assumed to be in the range $X \in(-1,1)$. An estimate of the input is typically obtained by low-pass filtering the output sequence $y(n)$. The state space equations describing the dynamics of the encoder shown in Fig. 1 are

$$
\begin{aligned}
{\left[\begin{array}{l}
u(n+1) \\
v(n+1)
\end{array}\right] } & =\mathbf{F}\left[\begin{array}{l}
u(n) \\
v(n)
\end{array}\right]+\vec{g} Q(u(n))+\vec{h} \\
& =\vec{f}(u(n), v(n), X)
\end{aligned}
$$

where $\mathbf{F}=\left[\begin{array}{cc}p_{2} & p_{1} \\ 0 & p_{1}\end{array}\right], \vec{g}=\left[\begin{array}{ll}-2, & -1\end{array}\right]^{T}, \vec{h}=\left[\begin{array}{ll}X, X \\ ]^{T} \text { and } T\end{array}\right.$ denotes the transpose operation. The term $\vec{g} Q(u(n))$ in (1) is $+\vec{g}$ or $-\vec{g}$ depending on the sign of $u$. This translates into dividing the $u-v$ phase-plane into two regions determined by the sign of $u(n)$, with the switching line, $S_{0}$, defined by the line $u=0$. On each side of $S_{0}$ the state space equations are Linear and Time-Invariant (LTI), with eigenvalues at $p_{1}$ and $p_{2}$. Traditionally, $\Sigma \Delta$ modulators are built and analyzed assuming ideal integrators or equivalently assuming that the open loop poles are on the unit circle. However, in practice circuit nonidealities result in imperfect or leaky integrations, i.e., $\left|p_{1}\right|<1$ and $\left|p_{2}\right|<1$ [4]. Tones in these modulators are attributed to the states being locked in a periodic orbit [13].

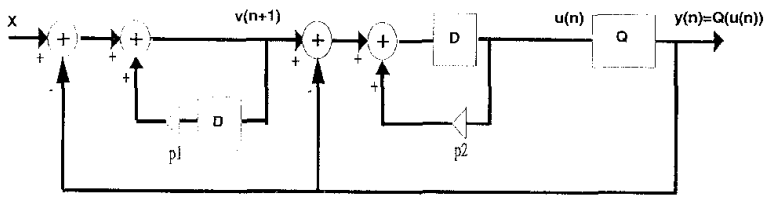

Fig. 1. Double loop $\Sigma \Delta$ modulator.

When either of the poles of the system in Fig. 1 are outside the unit circle, $\left|p_{1}\right|>1$ and/or $\left|p_{2}\right|>1$, all periodic orbits of the system become unstable ${ }^{3}$; thus precluding spectral peaks caused by stable periodic motion in the state space. The steady state behavior for this case is analyzed in Sections III and IV, while the tone behavior is studied in Section VI. In all that follows, we assume that both $p_{1}$ and $p_{2}$ are outside the unit circle. However, our analysis may be applied when only one of the integrator poles is moved outside the unit circle while the other is kept inside the unit circle.

\section{UNBOUNDEDNESS BOUNDARY}

One of the main side effects of moving the open loop poles outside the unit circle is the possibility of state trajectories becoming unbounded. For instance, it is easy to check via computer simulations, that the states of the double loop $\Sigma \Delta$ modulator with poles at 1.02 and zero initial conditions are bounded for a constant input of 0.4 , and become unbounded when the input is increased to $X=0.9$. In this section, we derive a system of continuous time differential equations that approximates the behavior of the states of the double loop modulator. We then characterize the steady state behavior of the continuous time system and prove that when the continuous time system exhibits an unstable limit cycle, the limit cycle provides us with a saturation measure on the states of the double loop modulator. A similar approach was proposed in [14] to obtain saturation bounds for the double loop modulator with poles on the unit circle. Other references on characterizing stability boundaries for the double loop $\Sigma \Delta$ modulator include [15] and [16]. Reference [15] determines bounds on the states of the double loop modulator with poles on the unit circle and $\mathrm{dc}$ inputs, while [16] determines bounds on the internal states of the double loop modulator with zero dc input and poles outside the unit circle.

Here, it is important to distinguish between limit cycle instability and encoder saturation ${ }^{4}$. While limit cycle instability is determined by the behavior due to small perturbations in the state trajectory, encoder saturation is determined by the magnitude of the internal variables of the system. Specifically, the encoder becomes saturated if the states of the integrators become excessively large or unbounded.

${ }^{3}$ To see this, note that the nonlinearity, $Q(\cdot)$, in system (1) is insensitive to small perturbations of the trajectories. Hence, the overall stability of limit cycles of the system is governed by powers of the eigenvalues of the open loop system. This result first appeared in Bulzacchelli, Chaos in Oversampled Analog-to-Digital Convertors, Area Exam Report, Massachusetts Institute of Technology, April 1992.

${ }^{4}$ Note that encoder saturation is at times referred to as encoder stability in [12]. 


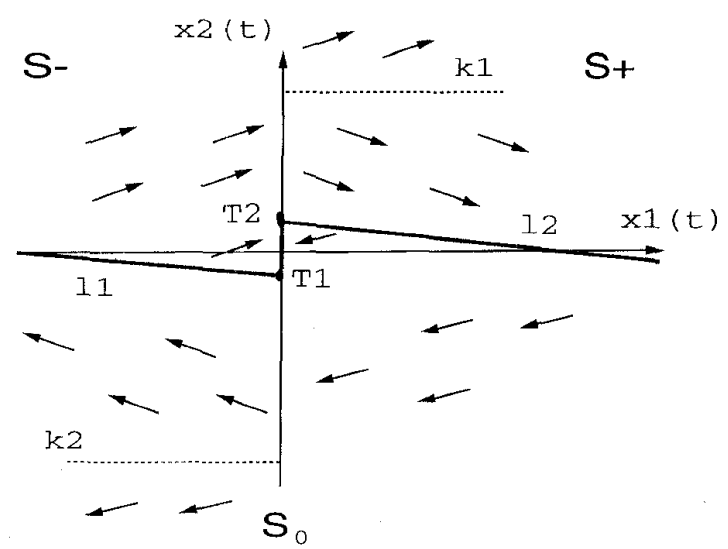

Fig. 2. Typical direction of flow of the continuous time vector fields.

\section{A. Geometric Analysis of an Associated Continuous Time System}

Let $S_{-}$and $S_{+}$in Fig. 2 denote the regions in space divided by the switching line, $S_{0}$ as defined in Section II. On each side of $S_{0}$, the discrete time trajectories may be thought of as samples of smooth continuous time trajectories. Equivalently, if we consider the discrete time system on each side of $S_{0}$, as a sampled-data model [17] for a continuous time system, then we can associate a LTI continuous time system of differential equations with the discrete time trajectories of (1)

$$
\begin{aligned}
\frac{d}{d t} \vec{x}(t) & =\mathbf{A} \vec{x}(t)+\vec{b} s+\vec{c} \\
& =\vec{f}_{ \pm}^{c}(\vec{x})
\end{aligned}
$$

where $\vec{x}$ contains the states of the continuous time system, $\vec{x}=\left[x_{1}, x_{2}\right]^{T}, \mathbf{A}=\log (\mathbf{F}), \vec{b} s=(\mathbf{F}-\mathbf{I})^{-1} \log (\mathbf{F}) \vec{g} s, \vec{c}=$ $(\mathbf{F}-\mathbf{I})^{-1} \log (\mathbf{F}) \vec{h}, \mathbf{I}$ denotes the identity matrix, $s=+1$ when $x_{1}>0$, and $s=-1$ when $x_{1} \leq 0^{5} . \vec{f}_{+}^{c}(\vec{x})$ denotes the vector field in $S_{+}$, while $\vec{f} \stackrel{-}{c}(\vec{x})$ denotes the vector field in $S_{-}$. Given the continuous time system (2), the discrete time system matrix, $\mathbf{F}$, is obtained by taking the matrix exponential of $\mathbf{A}$, i.e., $\mathbf{F}=e^{\mathbf{A} T}$. Sampling the trajectories defined by (2), with sampling rate $T=1$, results in the discrete time trajectories of the $\Sigma \Delta$ modulator, provided the switching boundary $S_{0}$ is not crossed.

Equation (2) describes a piecewise continuous differential equation with the right-hand side (RHS) discontinuous across $S_{0}$. On each side of $S_{0}$, the solutions to the differential equations are unique and smooth curves in $\Re^{2}$. The solution

${ }^{5} \log (\mathbf{F})$ is computed in terms of the values of $\log (\cdot)$ on the spectrum of $F$ [18]. $\log (\cdot)$ is a nonunique function, however, since the underlying discrete time system has real eigenvalues, we use the solution to $\log (\mathbf{F})$ that possesses eigenvalues on the real axis. Thus, for $p_{1}=p_{2}=p$ the values of $\mathbf{A}, \vec{b}$ and $\vec{c}$ are

$$
\begin{aligned}
\mathbf{A} & =\left[\begin{array}{cc}
\log (p) & 1 \\
0 & \log (p)
\end{array}\right] \quad \vec{b}=\frac{1}{(p-1)^{2}}\left[\begin{array}{c}
\log (p)(2-p)-(p-1) \\
-(p-1) \log (p)
\end{array}\right] \\
\text { and } & \quad \vec{c}=\frac{1}{(p-1)^{2}}\left[\begin{array}{c}
(p-1) X-X \log (p) \\
X(p-1) \log (p)
\end{array}\right]
\end{aligned}
$$

provided that $\log (p)$ is defined. Similar expressions are obtained when $p_{1} \neq p_{2}$. concept for differential equations with discontinuous RHS was proposed by Filippov [19]. Below, we give an intuitive description of the dynamics at $S_{0}$ by examining the directions of the vector fields on each side of the discontinuity, for a formal treatment refer to [19]. Fig. 2 illustrates a typical flow diagram for the continuous trajectories of (2) with both poles outside the unit circle. The arrows show the direction of flow of the continuous time trajectories. The solid rays denoted by 11 , and 12 are the boundary about which $\frac{d}{d t} x_{1}$ changes sign in $S_{-}$, and $S_{+}$, respectively,

and

$$
l 1:\left\{x_{1}, x_{2} \mid x_{1}=\frac{+b_{1}-c_{1}-a_{12} x_{2}}{a_{11}}\right\}
$$

$$
l 2:\left\{x_{1}, x_{2} \mid x_{1}=\frac{-b_{1}-c_{1}-a_{12} x_{2}}{a_{11}}\right\} .
$$

Similarly, the dashed rays denoted by $k 1$ and $k 2$ are the boundary about which the vector field changes direction in the $x_{2}$ direction in $S_{+}$and $S_{-}$respectively,

$$
k 1:\left\{x_{1}, x_{2} \mid x_{2}=\frac{-b_{2}-c_{2}}{a_{22}}\right\}
$$

and

$$
k 2:\left\{x_{1}, x_{2} \mid x_{2}=\frac{b_{2}-c_{2}}{a_{22}}\right\}
$$

where $a_{i j}, b_{i}$, and $c_{i}$ denote the respective elements of $\mathbf{A}, \vec{b}$, and $\vec{c}$. Note that, as $p_{1} \rightarrow 1$, the $x_{2}$ intercepts of $k 1$ and $k 2$ tend to $+\infty$ and $-\infty$, respectively.

Points $T 1$ and $T 2$ in Fig. 2 denote the $x_{2}$ intercept of $l 1$ and $l 2$, respectively. Near the switching line with $x_{2}>T 2$, the trajectories in the left-half plane point toward $S_{0}$, while those in the right-half plane point away from $S_{0}$. Thus, the solution to the differential equations is continued across the discontinuity in this region. Similarly, the solutions to the differential equations are continued through the discontinuity for $x_{2}<T 1$. In the region on the $x_{2}$-axis between $T 1$ and $T 2$, however, the trajectories on both sides of $S_{0}$ point toward the discontinuity. Intuitively, imperfections of the switching mechanism cause the state trajectories in this region to chatter on the switching line. This stable chattering motion converges toward an equilibrium point of an averaged model of the system $^{6}$ [19]. The direction of the vector field in $S_{+}$above $k 1$, and in $S_{-}$below $k 2$ imply that once a continuous time trajectory is in either of these regions, it will grow to infinity.

The above discussion points to the possibility of two types of asymptotic behavior in the continuous time state space, one

${ }^{6}$ The switching boundary in system (2) is defined by $S_{0}=\left\{x_{1}, x_{2} \mid\right.$ $\left.x_{1}=0\right\}$. Let $\lambda_{+}(\vec{x})=\frac{d x_{1}(t)}{d t}$ for $\vec{x} \in S_{+}$and $\lambda_{-}(\vec{x})=\frac{d x_{1}(t)}{d t}$ for $\vec{x} \in S_{-} \cdot \lambda_{+}(\vec{x})$ and $\lambda_{-}(\vec{x})$ denote the rate of change of $S_{0}$ along the trajectories of $\vec{f}_{+}^{c}(\vec{x})$ and $\vec{f}_{-}^{c}(\vec{x})$, respectively. Let $\vec{x}_{0}$ be a point on the switching boundary between $T 1$ and $T 2$, then, $\lambda_{+}\left(\vec{x}_{0}\right)=\lim _{\vec{x} \rightarrow \vec{x}_{0}} \lambda_{+}(\vec{x})$, and $\lambda_{-}\left(\vec{x}_{0}\right)=\lim _{\vec{x} \rightarrow \vec{x}_{0}} \lambda_{-}(\vec{x})$. The direction of the vector field on either side of the $x_{2}$-axis between $T 1$ and $T 2$ in Fig. 2 translates into having $\lambda_{+}\left(\vec{x}_{0}\right)<0$ and $\lambda_{-}\left(\vec{x}_{0}\right)>0$. By Lemma 3 of [19], the dynamics on $S_{0}$ between $T 1$ and $T 2$ are governed by an average value between $\vec{f}_{+}^{C}(\cdot)$ and $\vec{f}_{-}^{c}(\cdot), \frac{d}{d t} \vec{x}_{0}(t)=\vec{f}_{\mathrm{avg}}\left(\vec{x}_{0}\right)=\frac{\lambda_{-}\left(\vec{x}_{0}\right)}{\lambda_{+}\left(\vec{x}_{0}\right)-\lambda_{-}\left(\vec{x}_{0}\right)} \vec{f}_{+}^{c}\left(\vec{x}_{0}\right)+$ $\frac{\lambda_{+}\left(\vec{x}_{0}\right)}{\lambda_{+}\left(\vec{x}_{0}\right)-\lambda_{-}\left(\vec{x}_{0}\right)} \vec{f}_{-}^{c}\left(\vec{x}_{0}\right)$. The equilibrium point, $\vec{x}_{0}$, of this averaged dynamies is obtained by solving $\vec{f}_{\mathrm{avg}}\left(\bar{x}_{0}\right)=0$ for $\bar{x}_{0}, \bar{x}_{0}=\left[\frac{c_{1} b_{2}-b_{1} c_{2}}{b_{1} a_{22}-a_{1} b_{2}}\right]$. 
characterized by stable chattering motion on the switching line, and the other by the states growing to infinity.

Proposition 1: When system (2) exhibits a chattering region and a region where the states go unbounded, then system (2) also exhibits an unstable limit cycle outside of which continuous time trajectories grow to infinity.

(See the Appendix for proof.)

In all that follows, we refer to this unstable limit cycle as the unstable boundary limit cycle or the unboundedness boundary. A limit cycle of system (2) is obtained when a trajectory $\Gamma_{-}$ in $S_{-}$intersects a trajectory $\Gamma_{+}$in $S_{+}$at two points on the switching line. Note that since we consider a system with real poles, $\Gamma_{-}$and $\Gamma_{+}$can intersect at, at most 2 points [20]. Uniqueness properties ${ }^{7}$ of LTI differential equations in $\Re^{2}$ imply that all continuous time trajectories starting inside the unstable boundary limit cycle always remain inside, while trajectories starting outside the unstable limit cycle will remain outside. Furthermore, from Proposition 1, trajectories starting outside the unstable boundary limit cycle will go to infinity.

\section{B. Boundedness of the Discrete Time System}

On either side of $S_{0}$, the discrete time trajectories follow the direction of flow of the vector field of the continuous time system. Therefore, the continuous time trajectories trace through the discrete time trajectories as long as the switching line is not crossed. The main difference between the continuous time and the discrete time systems can be explained by noting that the differential equations have continuous solutions through the switching line $\left(x_{1}=0\right)$ outside of $x_{2} \in\left[T_{1}, T_{2}\right]$. The discrete time trajectories, however, will in general jump over the switching line and follow a different orbit than the continuous time system. In this subsection we prove that when a discrete time trajectory is outside the unstable boundary limit cycle, it will remain outside this boundary and become unbounded.

Recall from Section 3.1 that the arcs in $S_{-}$and $S_{+}$defining the unstable boundary limit cycle intersect on the switching line. Denote these arcs along with their continuation through the switching line by $\Gamma_{-}$and $\Gamma_{+}$, as shown in Fig. 3. $\Gamma_{-}$in Fig. 3 is a solution to the differential equation, $\vec{f}_{-}^{c}(\vec{x})$, in $S_{-}$, where $\vec{f}_{-}^{c}(\vec{x})$ is applied even when $\Gamma_{-}$is in $S_{+}$. Similarly, $\Gamma_{+}$is a solution to $\vec{f}_{+}^{c}(\vec{x})$, where $\vec{f}_{+}^{c}(\vec{x})$ is applied even when $\Gamma_{-}$is in $S_{+}$. The portion of $\Gamma_{-}$that is strictly in $S_{-}$along with the portion of $\Gamma_{+}$that is strictly in $S_{+}$constitute the unstable boundary limit cycle. From the uniqueness properties ${ }^{8}$ of ordinary differential equations in $\Re^{2}$, we deduce that as a discrete time trajectory outside of the unstable boundary limit cycle crosses the switching line from $S_{-}$to $S_{+}$, it will stay above arc $\Gamma_{-}$before following the direction of vector field in $S_{+}$; hence, it will remain outside the boundary defined by the

\footnotetext{
${ }^{7}$ Here, we are referring to uniqueness of solutions of LTI systems of differential equations in $R^{2}$.

${ }^{8}$ Specifically, continuous time trajectories following the direction of $\vec{f}_{+}^{c}(\vec{x})\left(\vec{f}_{-}^{c}(\vec{x})\right)$ cannot intersect. Since the discrete time trajectories in $S_{+}\left(S_{-}\right)$are samples of the continuous time trajectories in $S_{+}\left(S_{-}\right)$, a discrete time trajectory in $S_{+}\left(S_{-}\right)$cannot be in such a way that its corresponding continuous time trajectory would cross another continuous time trajectory following the direction of the vector field in $S_{+}\left(S_{-}\right)$.
}

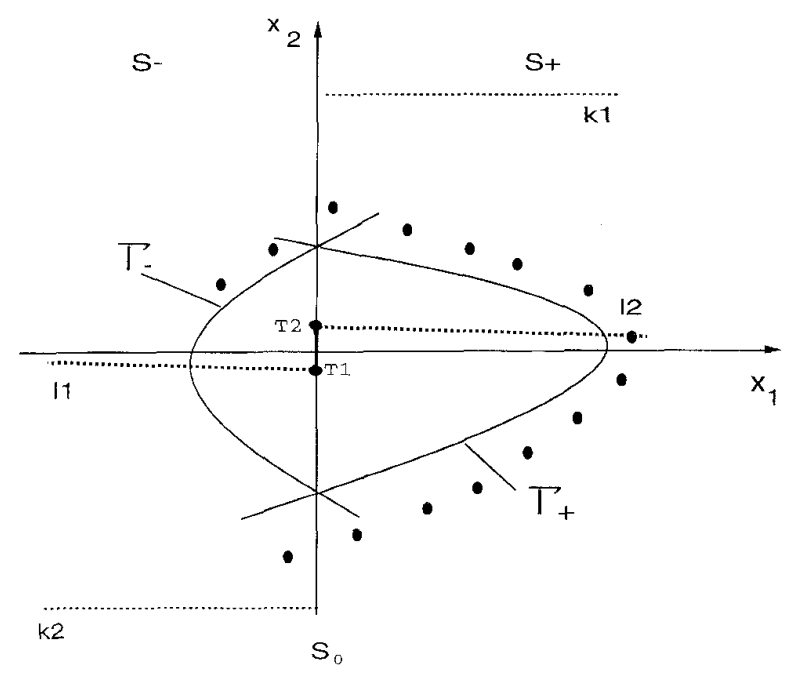

Fig. 3. Unboundedness boundary.

unstable limit cycle. Similarly a discrete time trajectory outside of the unstable boundary limit cycle crossing the switching line from $S_{+}$to $S_{-}$will stay below arc $\Gamma_{+}$, and will remain outside the boundary. A typical discrete time trajectory outside of the unstable boundary limit cycle is shown by the dots in Fig. 3. It is important to note that, discrete time trajectories inside the unstable boundary limit cycle may jump outside the boundary defined by the unstable limit cycle as they cross the switching line, however once such a trajectory is outside the boundary, it will remain outside. Furthermore, since discrete time trajectories follow the direction of the vector field of continuous time trajectories, a discrete time trajectory outside the unstable boundary limit cycle grows to infinity. Thus, we can use the unstable boundary limit cycle to check for unboundedness of the discrete time trajectories.

Fig. 4 shows the steady state behavior of the discrete time double loop $\Sigma \Delta$ modulator with both poles at 1.02 , a constant input of $X=0.4$, and two different initial conditions. The closed orbit in this figure corresponds to the unstable boundary limit cycle of the associated continuous time system. For an initial condition at $u(0)=0.5$, and $v(0)=7.5$, the trajectory converges to a region near the center of the $u-v$ plane. Once in this region, it appears to follow a random pattern. The trajectories near the center of the $u-v$ plane are globally bounded. Recall that a pole outside the unit circle precludes the existence of stable periodic trajectories. From the definition of a strange attractor ${ }^{9}$ in [21], the region near the center of the $u-v$ plane in Fig. 4 appears to behave as an attractor. When the initial condition is moved to $u(0)=0$, and $v(0)=7.5$, the discrete time trajectory jumps over the unstable boundary limit cycle after 1 time step, and becomes unbounded as $n \rightarrow \infty$.

\section{TRAPPING REgION}

The continuous time trajectories of system (2) reach the switching line before experiencing a change in the direction of vector field. The discrete time trajectories of the double $\Sigma \Delta$

${ }^{9}$ A strange attractor is a region in state space to which chaotic trajectories converge [21] 


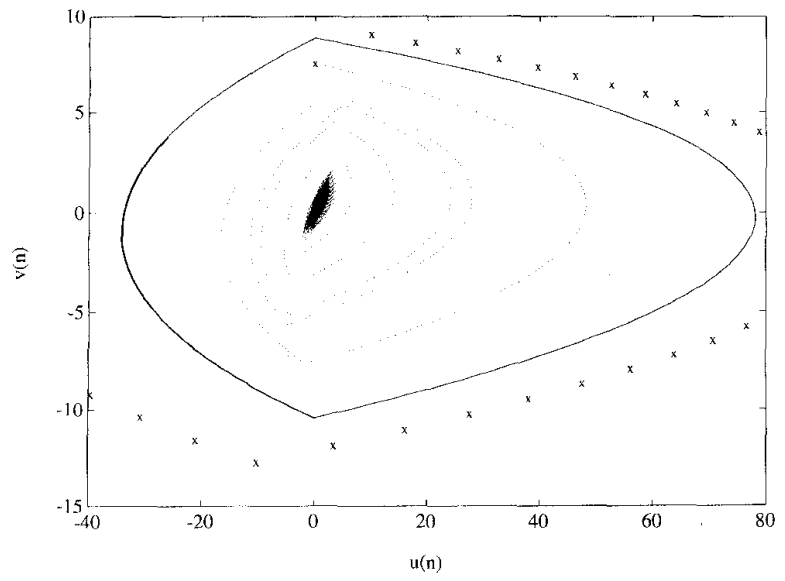

Fig. 4. State Space Trajectories, $\cdots$ : i.c. $=(0.5,7.5), \times \times$ : i.c. $=(0,7.5)$.

modulator, however, take discrete steps and hence jump over the switching line before changing direction of vector field. In this section we determine how far a discrete time trajectory can jump over the switching line and utilize this information to derive a second continuous time approximation for the discrete time modulator. The properties of this second continuous time system provide a bound on maximum signal levels.

\section{A. Behavior of the Discrete Time System About the Switching Line}

Let $m 1$ be the ray obtained by solving (1) subject to $u(n)=0$ and $u(n+1)>0, m 1:\{u, v \mid u>0$ and $v=u-1\}$, and $R 1$ correspond to the region in $u-v$ plane that lies above $m 1, R 1:\{u, v \mid u>0$ and $v \geq u-1\}$. Similarly, let $m 2$ be the ray obtained by solving (1) subject to $u(n)=0^{+10}$ and $u(n+1)<0, m 2:\{u, v \mid u<0$ and $v=u+1\}$, and $R 2$ denote the region below $m 2$ in the $u-v$ plane $R 2:\{u, v \mid u<0$ and $v \leq u+1\}$, as shown in Fig. 5 .

Proposition 2: A discrete time trajectory following the direction of the vector field in $S_{-}\left(S_{+}\right)$must land in region $R 1(R 2)$ as it crosses the switching line, at which point it will follow the direction of the vector field in $S_{+}\left(S_{-}\right)$.

Proof: In order to prove Proposition 2 for trajectories crossing the switching line from $S_{-}$to $S_{+}$we need to show that $\forall(u(n), v(n))$ such that $u(n) \leq 0$ and $u(n+1)>$ $0,(u(n+1), v(n+1))$ lies in region $R 1$, or, equivalently, $v(n+1)-u(n+1)+1 \geq 0$. Substituting in for $v(n+1)$, and $u(n+1)$ from Eq. (1) we get

$$
\begin{aligned}
v(n+1)-u(n+1)+1= & p_{1} v(n)-Q(u(n))+X-p_{2} u(n) \\
& -p_{1} v(n)+2 Q\left(u_{n}\right)-X+1 \\
= & -p_{2} u(n) \geq 0
\end{aligned}
$$

with equality achieved when $u(n)=0$.

The proof is similar for trajectories crossing the switching line from $S_{+}$to $S_{-}$. Line $m 1(m 2)$ provides a bound on the jumps that discrete time trajectories make as they cross from $S_{-}\left(S_{+}\right)$to $S_{+}\left(S_{-}\right)$.

\footnotetext{
${ }^{10} u=0^{+}$denotes the line $\{u, v \mid u=\delta\}$, where $\delta$ is an infinitesimal positive number.
}

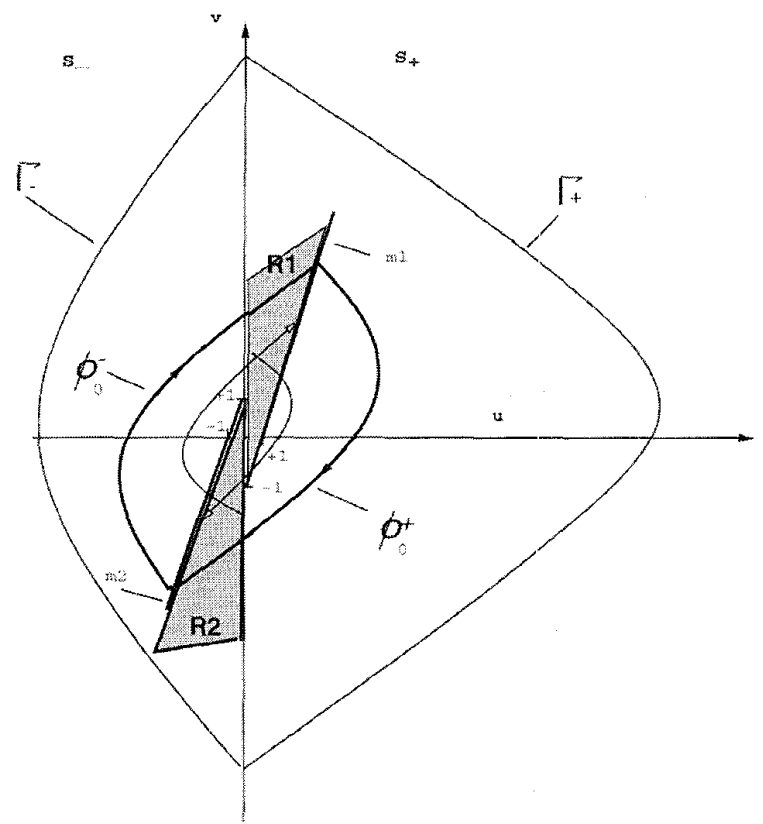

Fig. 5. Jumping effect of the discrete time system.

\section{B. Continuous Time System with Hysteresis}

In this subsection, we modify the continuous time approximation of Section III to incorporate the jumping effect of discrete time trajectories and show that a limit cycle of the modified continuous time system defines a trapping region for the discrete time trajectories. The "worst case jumping effect" of the discrete time trajectories is captured by imposing the following hysteretic switching mechanism on the continuous time approximation: continuous time trajectories following the direction of $\vec{f}_{-}^{c}(\vec{x})$ are forced to change direction of vector field on line $m 1$, and continuous time trajectories following the direction of the vector field, $\vec{f}_{+}^{c}(\vec{x})$, are forced to change direction of vector field on line $m 2$. The continuous time system with this switching logic approximates a discrete time modulator whose trajectories always reach the line $u=0$.

A limit cycle of the continuous time system with hysteresis is obtained by searching for trajectories of $\vec{f}_{+}^{c}(\vec{x})$ and of $\vec{f}_{-}^{c}(\vec{x})$ that intersect on lines $m 1$ and $m 2$. A typical limit cycle of the continuous time system with hysteresis is illustrated by arcs $\phi_{o}^{+}$and $\phi_{o}^{-}$in Fig. 5. The results developed in Section 4.1 along with uniqueness of solutions to ordinary differential equations in $\Re^{2}$ imply that a limit cycle of the continuous time system with hysteresis defines a trapping region for the discrete time trajectories. To see this, note that as a discrete time trajectory inside the limit cycle of this modified continuous time system crosses the switching line from $S_{-}$to $S_{+}$, it will land in the shaded region $R 1$ below arc $\phi_{o}^{-}$before following the direction of the vector field in $S_{+}$, hence, it will remain inside the boundary defined by the limit cycle of the modified continuous time system. Similarly, a discrete time trajectory inside this limit cycle crossing the switching line from $S_{+}$to $S_{-}$will land in the shaded region $R 2$ above arc $\phi_{o}^{+}$, and will remain inside the boundary of the closed orbit. 


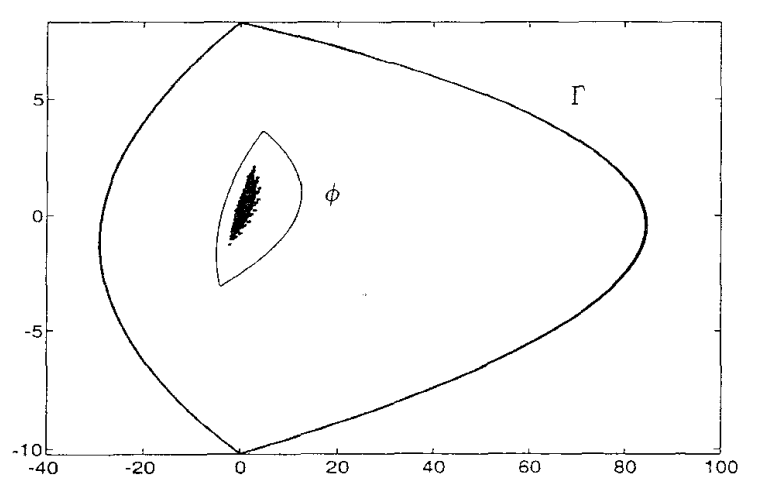

Fig. 6. Trapping region and unboundedness boundary for $p_{1}=p_{2}=1.02$, and $X=0.5$.

TABLE I

Maximum Allowable Input $X$ for a Grven $p_{1}=p_{2}$

\begin{tabular}{cc}
\hline$p_{1}=p_{2}$ & $X$ \\
\hline 1.02 & 0.8 \\
1.03 & 0.8 \\
1.04 & 0.7 \\
1.06 & 0.5 \\
1.08 & 0.2 \\
1.09 & 0 \\
\hline
\end{tabular}

Therefore, a limit cycle of the continuous time system with hysteresis provides a trapping region or boundedness boundary for discrete time trajectories. We note that it is possible for a discrete time trajectory outside the trapping region to cross to the inside as it jumps over the switching line. However, once inside, the trajectory will remain inside and will exhibit unstable but bounded behavior. Thus, all initial conditions inside the trapping region result in bounded behavior.

Recall that discrete time trajectories outside the unboundedness boundary defined in Section III remain outside this boundary and grow to infinity. This implies that the trapping region will always be inside the unboundedness boundary. In fact, the unboundedness boundary contains the basin of attraction of the discrete time attractor, which itself contains the trapping region. Fig. 6 shows the stability boundaries for $p_{1}=p_{2}=1.02$ and $X=0.5$. The closed orbits denoted by $\phi$ and $\Gamma$ in Fig. 6 correspond to the trapping region and unboundedness boundary, respectively. Also shown in the same figure is the discrete time attractor.

\section{INPUT DyNAMIC RANGE AND SNR PERFormanCE}

In this section we consider the performance of the double loop $\Sigma \Delta$ modulator with poles outside the unit circle. We begin by obtaining numerical results on the largest positive input values for which the states of the $\Sigma \Delta$ modulator remain bounded for a given pole location. These results are presented in Table I. The first column in Table I corresponds to the pole location, and the second to the largest constant input, to within \pm 0.1 , that remain bounded for 16000 time steps. Hence, we can conclude that as the poles of the double loop modulator move outside the unit circle, the maximum de value that does not result in saturation decreases.

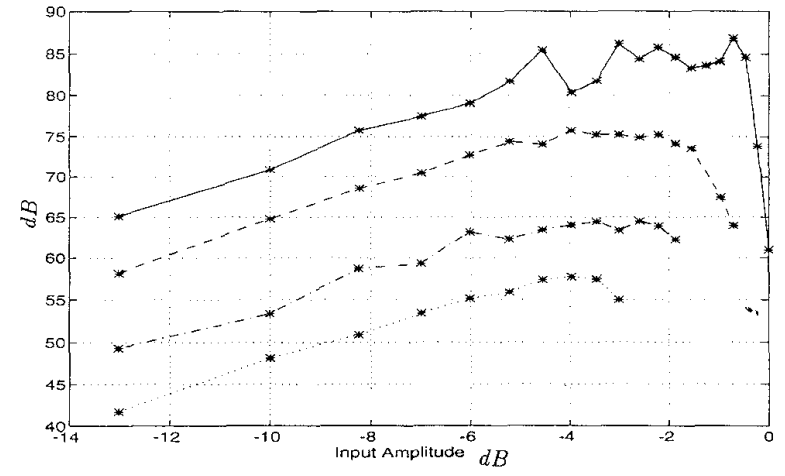

Fig. 7. Signal-to-noise ratio versus input amplitude, top curve: $p=p_{1}=p_{2}=1$, curve below: $p=1.02$, curve below: $p=1.04$, curve below: $p=1.06$.

Moving the poles outside the unit circle not only reduces the dynamic range but also affects the noise shaping characteristics of the $\Sigma \Delta$ modulator. Fig. 7 indicates that the SNR performance of the double loop modulator degenerates as the poles move further away from the unit circle. This figure is obtained by using a sinusoidal input with frequency at half the baseband and oversampling ratio of 128 .

\section{TONE BEHAVIOR}

When a limit cycle is asymptotically stable, periodicity occurs because the states $(u, v)$ move from one point on the limit cycle to another in a periodic fashion, resulting in tones. Moving the poles of the $\Sigma \Delta$ modulator outside the unit circle destabilizes any limit cycles thus preventing tones due to stable periodic motion of the state trajectories. While this approach improves the tone behavior, it does not completely remove idle tones. Consider the periodogram of the double loop $\Sigma \Delta$ modulator with $X=0, p_{1}=p_{2}=1.01$, and initial conditions $(1.1051,0.7462)$, shown in Fig. 8 . This periodogram was obtained by averaging over 6 FFT's, each 65536 points long, of the output bit stream, and is plotted in the frequency range $[0, \pi]$. Observe that the periodogram in Fig. 8 is broadband, but also has discrete components indicating predominant frequencies of the output sequence with period 8 . Even though the idle tones in the periodogram are outside the signal baseband, there is no guarantee that tones in the modulator with unstable filter dynamics will always fall outside the baseband of interest with different inputs, initial conditions or pole values. It is therefore important to study the nature of tones in the modulator with unstable filter dynamics.

In this section we consider the tone behavior of double loop $\Sigma \Delta$ modulators whose steady state behavior is inside the trapping region. We will show that for some unstable limit cycles, periodicity and therefore tones occur because the state $(u, v)$ persistently moves from the neighborhood of one point on the limit cycle to the neighborhood of another point on the limit cycle. We refer to these neighborhoods as attractors ${ }^{11}$, and to their associated unstable limit cycles as dominant limit cycles. We will develop a technique to approximate the

\footnotetext{
${ }^{11}$ Trajectories appear to be chaotic within such an attractor.
} 


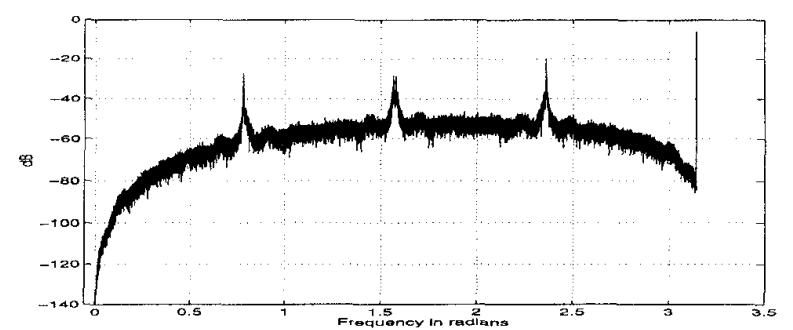

Fig. 8. Output periodogram in $\mathrm{dB}$ for $X=0, p_{1}=p_{2}=1.01$, initial conditions $=(1.1051,0.7462)$, and $w \in[0, \pi]$.

boundaries of these attractors. In all that follows, we assume that the limit cycles under consideration are generic, i.e., that no points on the limit cycles lie on the switching point of the quantizer.

\section{A. Small Signal Behavior}

In this subsection, we expand the approach in Section III-A and show that the local behavior of the state trajectories around different points on an unstable limit cycle can be described by continuous time LTI systems. Note that, we are only concerned with the unstable limit cycles that are inside the trapping region discussed in Section IV.

Given a limit cycle of period $N$ with corresponding $N$ bit sequence $s(\cdot)$, multiplying by appropriate powers of $F$ and summing (1) over the limit cycle we obtain ${ }^{12}$

$\left[\begin{array}{c}u(k) \\ v(k)\end{array}\right]=\left(\mathbf{I}-\mathbf{F}^{N}\right)^{-1}\left\{\sum_{i=1}^{N}\left\{\mathbf{F}^{N-i} \vec{h}+\mathbf{F}^{N-i} \vec{g} s(k+i-1)\right\}\right\}$

for $1 \leq k \leq N$, where $\mathbf{I}$ denotes the $2 \times 2$ identity matrix. Using (3) we can check for the existence of a given unstable limit cycle of period $N$ by Tsypkin's method [22]:

1) Select a candidate bit sequence $s(\cdot)$.

2) Solve (3) for $u(k)$ and $v(k), k=1, \cdots, N$.

3) The candidate limit cycle is a valid periodic orbit of the system if the polarities of $u(k)$, for $k=1, \cdots, N$, are consistent with the assumed bit sequence $s(\cdot)$.

Let $L$ denote an unstable limit cycle of period $N$ and $\vec{P}$ be a point on $L$. We denote by $s_{\vec{P}}(\cdot)$ the $N$ point sequence of +1 's and -1 's that, if substituted for $Q(u(\cdot))$ in (1), results in $\left[\begin{array}{l}u(k+N) \\ v(k+N)\end{array}\right]=\vec{P}$ when $\left[\begin{array}{l}u(k) \\ v(k)\end{array}\right]=\vec{P}$. Note that $\vec{P}$ is the equilibrium point of $f_{s_{\vec{P}}}^{N}(\cdot)$, the $N$ th sample point return map with $s_{\vec{P}}(\cdot)$ substituted for $Q(u(\cdot))$ in (1). Mathematically, $f_{s_{\vec{P}}}^{N}(\cdot)$ is given by

$$
\begin{aligned}
{\left[\begin{array}{l}
u_{\vec{P}}(n+1) \\
v_{\vec{P}}(n+1)
\end{array}\right] } & =\mathbf{F}^{N}\left[\begin{array}{l}
u_{\vec{P}}(n) \\
v_{\vec{P}}(n)
\end{array}\right]+\sum_{i=1}^{N} \mathbf{F}^{N-i}\left\{\vec{g} s_{\vec{P}}(i)+\vec{h}\right\} \\
& =f_{s_{\vec{P}}}^{N}\left(u_{\vec{P}}(n), v_{\vec{P}}(n)\right)
\end{aligned}
$$

\footnotetext{
${ }^{12}$ Equation (3) is obtained by assuming that $\{u(k), v(k)\}$ obtained by substituting $s(\cdot)$ for $Q(u(\cdot))$ in (1) is periodic with period $N$, that is, $u(k+N)=u(k)$ and $v(k+N)=v(k)$, and solving for $\{u(k), v(k)\}$ for $k=1, \cdots, N$.
}

Starting in a neighborhood of $\vec{P}$, and subsampling the trajectory of (1) by $N$, results in the trajectory of (4). Locally, the behavior in the neighborhood of $\vec{P}$ is determined by the eigenvalues and eigenvectors of the Jacobian of $f_{s_{\vec{P}}}^{N}$, i.e., $\mathbf{F}^{N}$.

Since $f_{s_{\vec{P}}}^{N}$ is LTI, we can further describe the local dynamics about $\vec{P}$, by considering the behavior of an associated continuous time system. Specifically, the discrete time trajectories defined by (4) may be thought of as samples of smooth continuous time trajectories. Consequently, we can associate a LTI continuous time system of differential equations with the discrete time trajectories of (4)

$$
\frac{d}{d t} \vec{x}_{s_{\vec{P}}}(t)=\mathbf{B} \vec{x}(t)+\vec{c}_{s_{\vec{P}}}
$$

where $\vec{x}_{\vec{P}_{\vec{P}}}$ contains the states of the continuous time system, $\vec{x}=\left[x_{1_{\vec{P}}}, x_{2_{\vec{p}}}\right]^{T}, \mathbf{B}=\log \left(\mathbf{F}^{N}\right)$, and $\vec{c}_{s_{\vec{P}}}=\left(\mathbf{F}^{N}-\right.$ I) ${ }^{-1} \mathbf{B} \sum_{i=1}^{N} \mathbf{F}^{N-i}\left\{\vec{g} s_{\vec{p}}(i)+\vec{h}\right\}$. We use the subscript $s_{\vec{P}}$ to stress that $(5)$ describes the local behavior about point $\vec{P}$ on the limit cycle. Note that, (5) has an equilibrium point at $\vec{P}$. For poles outside the unit circle with $p_{1} \neq p_{2}, \vec{P}$ corresponds to an unstable node, while for $p_{1}=p_{2}>1, \vec{P}$ corresponds to an unstable improper node [20].

The local descriptions in (4) and (5) are valid as long as bit sequence $s_{\vec{P}}$ describes the global behavior of the system correctly. For example, let $\vec{P}_{l}$ be the point closest to the switching line on the left of the switching line, i.e., the point on the unstable limit cycle that lies to the left of the switching line and whose distance from the switching line is less than that of all other points of the unstable limit cycle to the left of the switching line. Let $s_{\vec{P}_{l}}$ denote the corresponding bit sequence. Consider a local trajectory in the neighborhood of $\vec{P}_{l}$ that is moving away from $\vec{P}_{l}$ in the direction of the switching line. Once this local trajectory crosses the switching line, the dynamics governing the large signal behavior change since the sequence $s_{\vec{P}}$ is no longer the resulting bit pattern. Thus, as long as the switching line is not crossed by this local trajectory of $\vec{P}_{l}$, the global behavior is described by the trajectory jumping from the neighborhood of one point on the limit cycle to the neighborhood of another with (5) describing the local behavior about point $\vec{P}$ on the limit cycle.

The effect of the switching line on the local behavior about points on the unstable limit cycle translates into boundaries about each point on the limit cycle. Within these boundaries the local behavior about a point $\vec{P}_{i}$ on the limit cycle can be described by (4) or by the associated continuous time system of (5) with $s_{\vec{P}}$ substituted for $s_{\vec{P}}$. The switching line itself provides the left or right boundary for the points on the limit cycle that are closest to the switching line on the right or left of the switching line, respectively. For other points on the limit cycle, propagating the line $u=0^{-15}$ backward in time ${ }^{14}$

\footnotetext{
${ }^{13}$ Recall that $u=0^{-}$denotes the line $\{u, v \mid u=-\delta\}$, where $\delta$ is an infinitesimal positive number. Similarly, $u=0^{+}$denotes the line $\{u, v \mid u=+\delta\}$.

${ }^{14}$ Propagating the line $u=0^{-}$backward in time through (1) translates into $\left[\begin{array}{l}u(n) \\ v(n)\end{array}\right]=\mathbf{F}^{-1}\left[\begin{array}{l}u(n+1) \\ v(n+1)\end{array}\right]-\vec{g} s_{\vec{P}_{2}(N+n)}-\vec{h}$, for $n=0,-1, \cdots$. The sequence $s_{\vec{P}_{r}}(\cdot)$ is substituted in for $s_{\vec{P}_{l}}(\cdot)$ when propagating the line $u=0^{+}$ backward in time, where $\vec{P}_{T}$ denotes the point on the limit cycle closest to the switching line that lies to the right of the switching line.
} 
through (1), defines the bound to their right. Since $u=0^{-}$ is a straight line, this bound is also a straight line. Similarly, propagating the line $u=0^{+}$, backward in time through (1) defines the bound to the left of these points. This bound is also a straight line. In what follows, we refer to the bounds obtained by propagating the switching line backward in time through (1) as virtual switching lines. Since the rate of growth is the same for all points on the limit cycle, we only need to consider the local trajectories of points closest to the switching line on the left or right of the switching line.

As an illustrative example, consider an unstable limit cycle of the double loop modulator with $X=0$, and $p_{1}=p_{2}=$ 1.01. Using Tsypkin's method, we have found an unstable limit cycle of period $N=8$. The $x$ 's in Fig. 9(a) denote this unstable limit cycle, the directed arcs in the same figure describe the trajectory of the limit cycle, while the dashed vertical line denotes the switching line. For convenience we have labeled some of the points on the limit cycle. $\vec{P}_{1}$ is the point on the limit cycle that is closest to the switching line that lies to the left of the switching line. Motion in a neighborhood of $\vec{P}_{1}$ is defined by (4) with $s_{\vec{P}_{1}}(\cdot)=$ $(-1,1,1,-1,-1,1,-1,1)$. Fig. 9 (b) shows the associated continuous time trajectories around $\vec{P}_{1}$. This phase portrait is typical for unstable improper nodes. Fig. 9(b) was obtained by starting on points on the switching line and the virtual switching line $v_{l 1}$, and running (5) backward in time. As per our discussion in the previous paragraph, (4) with $s_{\vec{P}_{1}}(\cdot)$ is valid in describing the behavior of local trajectories that move to the right of $\vec{P}_{1}$, as long as the switching line is not crossed. Similarly, the behavior of the local trajectories that are repelled away from $\vec{P}_{1}$ to the left is correctly described by (4) with $s_{\vec{P}}(\cdot)=s_{\vec{P}_{1}}(\cdot)$ as long as the virtual switching line, $v_{l 1}$, is not crossed. The virtual switching line $v_{l 1}$ is shown as the solid line in Fig. 9(b).

\section{B. Large Signal Behavior: Discrete Time Attractors}

Given an initial condition in a neighborhood of an unstable limit cycle, the global behavior is temporarily described by the states $(u, v)$ moving from the neighborhood of a point on the unstable limit cycle to the neighborhood of another point on the limit cycle in a periodic fashion resulting in a periodic bit sequence at the output of the quantizer. This periodicity is interrupted once the local trajectory in the neighborhood of any point $\vec{P}$ on the limit cycle crosses the switching or virtual switching line. What is the global behavior once a local trajectory crosses the switching or virtual switching line? Will the states escape from the neighborhood of the unstable limit cycle altogether or will they get folded back to the neighborhood of the same unstable limit cycle? We will answer these questions for the unstable limit cycle of Fig. 9(a) by examining the local vector field about points $\vec{P}_{1}$ and $\vec{P}_{3}$ and show that at least in this particular example, the trajectory folds back rather than escapes. Throughout this section, we focus on the unstable limit cycle of Fig. 9(a) to illustrate our main points; however, the steps outlined below can be generalized to an arbitrary unstable limit cycle.
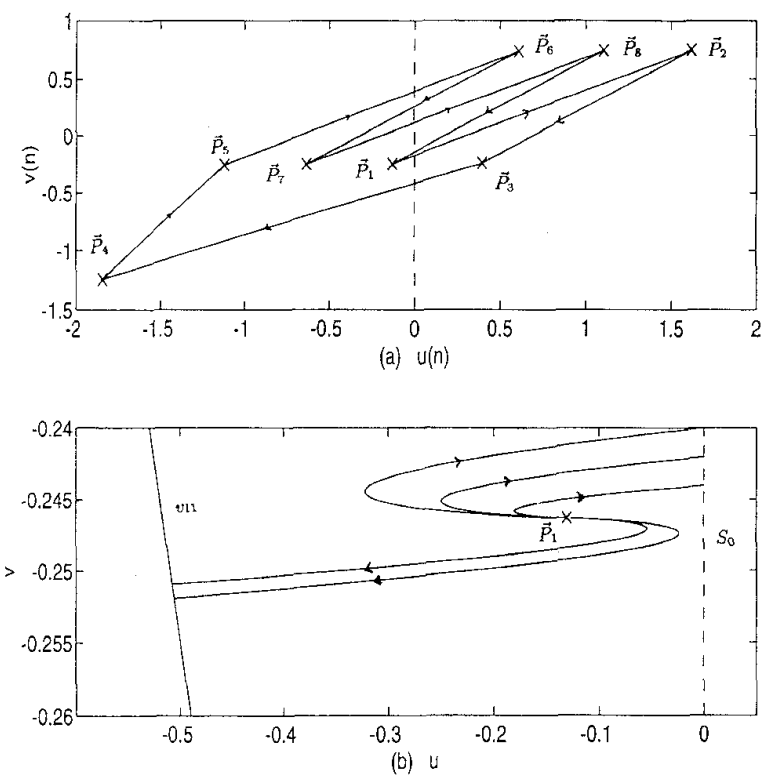

Fig. 9. $\mathrm{X}=0, p_{1}=p_{2}=1.01$; (a) Unstable limit cycle of period $N=8$. (b) Behavior of perturbations about point $\vec{P}_{1}$ on the limit cycle.

\section{Stability Analysis}

In this subsection, we will show that the unstable limit cycle of Fig. 9(a) has an attractor in its neighborhood and that within this attractor the steady state behavior is such that the bit sequence at the output of the quantizer is intermittently periodic, hence explaining the broadband character and the discrete components of the output periodogram shown in Fig. 8. Specifically, we will show that the bit sequence at the output of the quantizer is periodic except for occasional breaks in periodicity.

We begin by describing the local behavior in the neighborhoods of $\vec{P}_{1}$ and $\vec{P}_{3}{ }^{15}$ as shown in Fig. 10(a). Point $k 1$ in Fig. 10(a) defines the point on the switching line at which a local trajectory, $S_{3}$, in the neighborhood of $\vec{P}_{3}$ is tangent to the switching line ${ }^{16}$. Denote the corresponding trajectory about $\vec{P}_{1}$ by $S 1$. Below $x_{2}=k 1$ the direction of the vector field to the left of $P_{3}$ and to the right of the switching line points toward the switching line, while in the same region above $x_{2}=k 1$ the direction of the vector field points away from the switching line. Similarly, $k 2$ in Fig. 10(a) denotes the point on the switching line at which a local trajectory, $T 1$, in the neighborhood of $\vec{P}_{1}$ is tangent to the switching line. Arc $T 3$ denotes the local trajectory about $\vec{P}_{3}$ that corresponds to $T 1$. Below $x_{2}=k 2$ the direction of the vector field to the right of $P_{1}$ and to the left of the switching line points away from the switching line, while in the same region above $x_{2}=k 1$ the direction of the vector field points toward the switching line. In the region defined by $k 2<x_{2}<k 1$, the vector field immediately to the left and right the switching

\footnotetext{
${ }^{15}$ Note that $\vec{P}_{3}$ is two time steps shifted from $\vec{P}_{1}$,

${ }^{16}$ Here, the tangent curve is not unique to the limit cycle under consideration. Recall that for $p_{1}=p_{2}$, the equilibrium point of $(5)$ is an unstable improper node, thus the tangent curve will exist for points on any other limit cycle of the double loop modulator with $p_{1}=p_{2}$.
} 
line point toward the switching line. The motion along the switching line between points $k 1$ and $k 2$ can be characterized by a chattering motion along the switching line. Further analysis of the motion between points $k 1$ and $k 2$, by applying the averaging procedure in [19] as described in Section 3.1, shows that the averaged dynamics of the chattering motion has an unstable equilibrium point denoted by $E$ in Fig. 10(a). Consequently, local trajectories diverge from $E$ as they chatter along the switching line. Specifically, the direction of the vector field immediately to the left and to the right of the switching line above point $E$ is such that local trajectories move upward as they chatter along the switching line and eventually escape from the neighborhood of the unstable limit cycle. Below point $E$ in Fig. 10(a), the local trajectories move downward as they chatter along the switching line, until they are interjected to the left of arc $T 1^{17}$.

A local trajectory inside arc $T 1$ corresponds to a temporary behavior described by the states moving about points on the unstable limit cycle in a periodic fashion. Once the local trajectory to the left of $T 1$ crosses the virtual switching line $v_{l 1}$, the corresponding trajectory about $\vec{P}_{3}$ crosses the switching line and lands either to the left of $T 1$ or between $T 1$ and $L 1$, where $L 1$ denotes the local trajectory about $\vec{P}_{1}$ that passes through the point $E$ as shown in Fig. $10(a)^{18}$. The former again results in the periodic behavior described above; the latter results in a local trajectory that chatters downward along the switching line and eventually gets interjected back inside arc $T 1$, hence causing periodic behavior. Therefore, both in the former and latter cases the behavior of local trajectories is a succession of periodic behavior and chattering.

Putting these together we conclude that a trajectory starting between points $E$ and $k 2$ on the switching line will stay bounded in the neighborhood of the unstable limit cycle and will exhibit periodic behavior followed by chattering followed by periodic behavior. This results in an output bit sequence that is periodic except for occasional breaks in periodicity and is responsible for the peaks in the periodogram of Fig. 8.

We also conclude that the unstable limit cycle of Fig. 9(a) has an attractor in its neighborhood. Furthermore, the portion of the attractor about $\vec{P}_{1}$ is bounded below by the $\operatorname{arc} T 1$ and above by the arc $L 1$, as shown in Fig. $10(\mathrm{~b})$.

\section{Remarks}

The attractor about the unstable limit cycle of Fig. 9(a) consists of the union of the attracting regions about each point on the limit cycle, and is shown in Fig. 11. The $\times$ 's in this figure correspond to the unstable limit cycle of Fig. 9(a),

\footnotetext{
${ }^{17}$ To see that, we examine the direction of the vector field immediately to the left and to the right of the switching line. It can be shown that the direction of the vector field to the left of $\vec{P}_{3}$ and to the right of the switching line is such that the local trajectory of $\vec{P}_{3}$ at $k 2$ moves to the left of arc $T 1$. Furthermore, the discrete jumps that the local trajectories of $\vec{P}_{1}$ that are above arc $T 1$ make as they cross the switching line, fall above the line $v=k 2$. In fact in can be shown that these discrete jumps are bounded by a line corresponding to the image of the switching line under map (4) with $s_{\vec{P}}$ substituted for $s_{\vec{P}}$. This line intersects the switching line at point $v=k 2$. Thus local trajectories will eventually fall inside $T 1$ as they chatter between points $E$ and $k 2$.

${ }^{18}$ This is explained by noting that are $T 3$ crosses the switching line above point $k 2$.
}

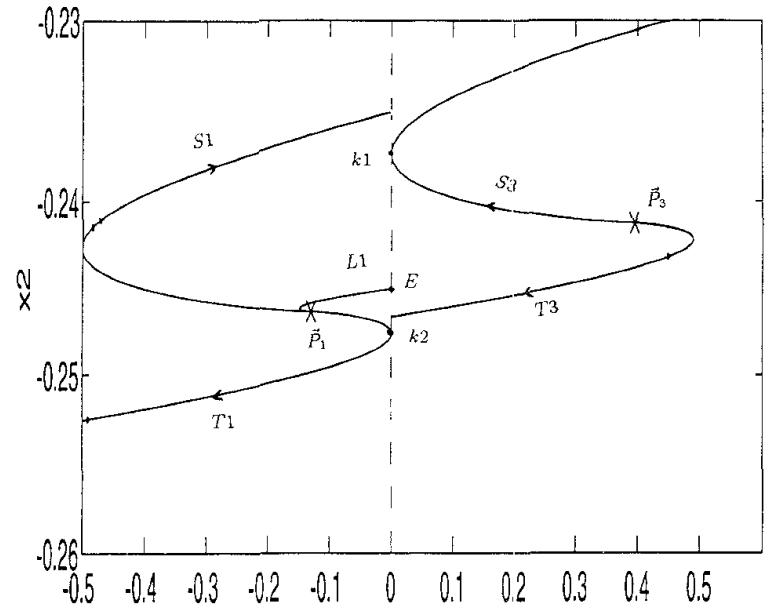

(a)

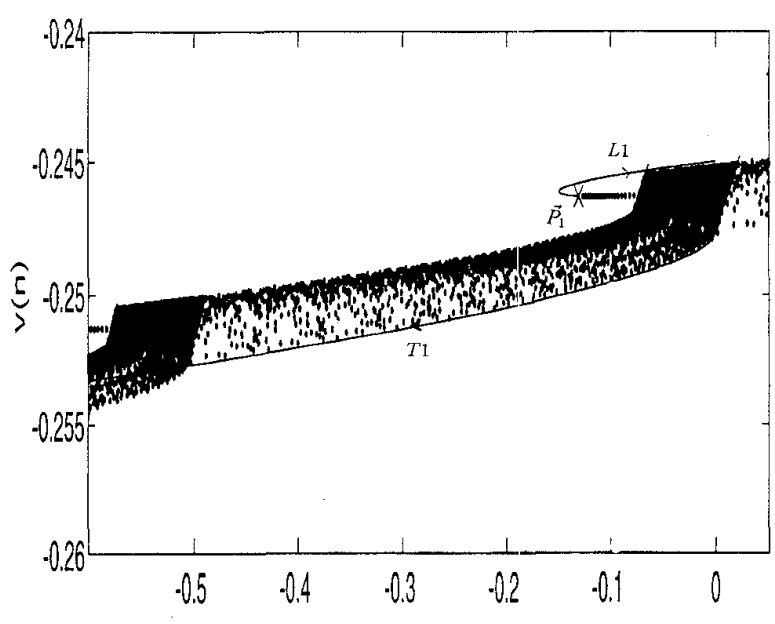

(b)

Fig. 10. (a) Local behavior about points on the unstable limit cycle. (b) Attractor about point $\vec{P}_{1}$.

and what appear to be lines going through the $x$ 's are actually the attractor itself ${ }^{19}$. As described earlier, the global behavior described by the states $(u, v)$ consists of moving from the neighborhood of one point on the limit cycle to the neighborhood of another point in a periodic fashion with occasional breaks in the periodicity. An estimate of the output periodogram corresponding to the state space trajectory shown in Fig. 11 is shown in Fig. 8. As expected, the spectral peaks are in agreement with the fact that the limit cycle has period 8 .

As the poles of the modulator are moved further out from the unit circle, the tonal content improves, however, the high frequency peaks persist. For instance, for the above example, the tone at $w=\pi$ in Fig. 8 persists as the poles are moved away from the unit circle. Finally, we note that it is possible

\footnotetext{
${ }^{19}$ Note that the attractor in Fig. 11 fills out a small dense portion of the state space in the neighborhood of the unstable limit cycle. This is typical of the attractors exhibited in the double loop modulator with zero input. Discrete time attractors for nonzero inputs, however, tend to fill out a larger portion of the state space.
} 


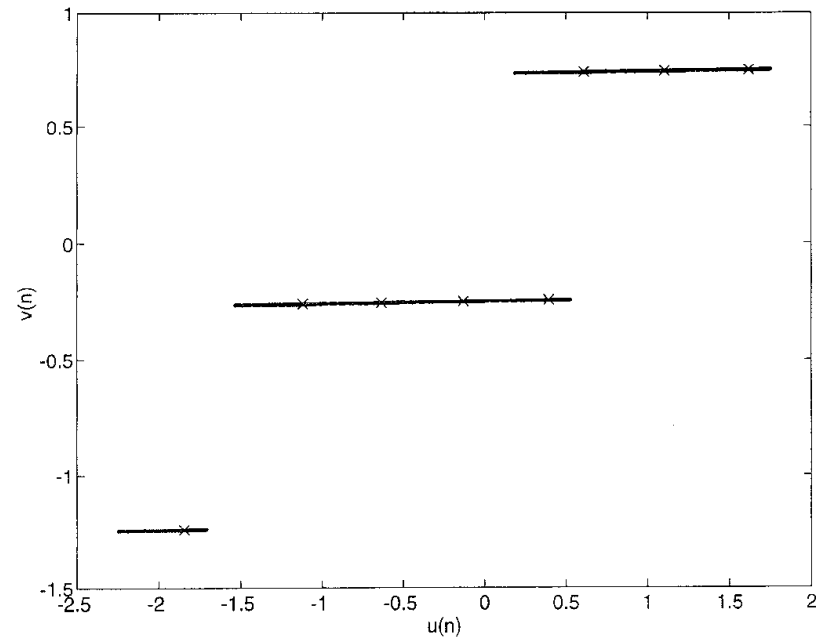

Fig. 11. Attractor about the unstable limit cycle of period $N=8$, for $X=$ 0, and $p_{1}=p_{2}=1.01$

for the state space trajectories to exhibit more complicated behavior that results in spectral peaks that are incommensurate.

\section{CONCLUDING REMARKS}

We have considered the saturation and tone behavior of the double loop $\Sigma \Delta$ modulator with unstable filter dynamics. We have shown that with poles outside the unit circle: (1) there is the possibility of the encoder becoming saturated, (2) the dynamic range of the encoder is reduced, (3) there is a loss in SNR. To avoid saturation, we studied two continuous time approximations to the discrete time modulator. The continuous time analysis provides us with an approximate stability boundary in the state space. We have also shown that while the tone behavior of the double loop $\Sigma \Delta$ modulator is improved when poles are moved outside the unit circle, not all spectral peaks are removed. We have explored the steady state behavior of the double loop modulator within its stability region, and have found that dominant unstable limit cycles determine repeating patterns in the state space and result in tones. We have presented a numerical method to determine which unstable limit cycle results in tones. Future direction of research is to uncover the exact relation between tonal strength and pole moduli, and to extend our results to the case where a dither signal is present.

\section{APPENDIX}

Proof of Proposition 1: Consider the flow diagram shown in Fig. 2. Let $S_{0_{1}}$ be the portion of the $x_{2}$-axis between the $x_{2}$ intercept of $k 1$ and the $x_{2}$-intercept of $l 2, S_{0_{1}}:\left\{\vec{x} \in \Re^{2} \mid x_{1}=\right.$ $\left.0, \frac{-b_{1}-c_{1}}{a_{12}} \leq x_{2} \leq \frac{-b_{2}-c_{2}}{a_{22}}\right\}$. There exists a subset, $O \subset S_{0_{1}}$, such that the trajectories of system (2) that emanate from $O$ following the direction of the vector field in $S_{+}$are returned to $S_{0_{1}}$ following the vector field in $S_{-}$. We prove proposition 1 by considering the Poincare map, $P: O \rightarrow S_{0_{1}}$, under the flow defined by system (2), and showing that $P(y)=y$ for at least one $y \in O$. This is equivalent to showing that $P(\cdot)$ has at least one fixed point in $O$ or that the underlying continuous time system (2) has at least one limit cycle that intercepts the $x_{2}$-axis in $O[21$, ch. 2]. The slope of $P(\cdot)$ at the point(s) where $P(y)=y$ determines the stability of the underlying limit cycle(s).

We derive the Poincare map for $p_{1}=p_{2}=p$, a similar expression is obtained when pole values are not equal. For trajectories of system (2) that emanate from $O$ following the direction of $\vec{f}_{+}^{c}(\vec{x})$ and return to $S_{0_{1}}$ following the of $\vec{f}_{-}^{c}(\vec{x})$, the first return map is

$$
\begin{aligned}
y(k+1)= & P(y(k)) \\
= & p^{\tau_{1}+\tau_{2}} y(k)+p^{\tau_{1}+\tau_{2}} \frac{1-X}{p-1} \\
& +p^{\tau^{2}} \frac{1-X}{p-1}-p^{\tau^{2}} \frac{1+X}{1-p}+\frac{1+X}{1-p}
\end{aligned}
$$

where $\tau_{1}$ is the time it takes for a trajectory emanating from $O$ to cross the $x_{2}$-axis at a point $w$ below the $x_{2}$-intercept of $l 1$, and $\tau_{2}$ is the time it takes for this trajectory to return to $S_{0_{1}}$ from $w$. Time variables, $\tau_{1}$ and $\tau_{2}$, are found by solving transcedental equations involving $y(k)$,

$$
-p^{\tau_{1}} \bar{x}_{1}^{+}+\tau_{1} p^{\tau_{1}}\left(y(k)-\bar{x}_{2}^{+}\right)+\bar{x}_{1}^{+}=0
$$

and

$$
\bar{x}_{1}^{-}-p^{\tau_{2}} \bar{x}_{1}^{-}+\tau_{2} p^{\tau_{2}}\left(p^{\tau_{1}}\left(y(k)-\bar{x}_{2}^{+}\right)+\bar{x}_{2}^{+}-\bar{x}_{2}^{-}\right)=0
$$

where $\overrightarrow{\vec{x}}^{+}=\left[\bar{x}_{1}^{+}, \bar{x}_{2}^{+}\right]^{T}=\mathbf{A}^{-1}(-\vec{b}-\vec{c})$ and $\vec{x}^{-}=$ $\left[\bar{x}_{1}^{-}, \bar{x}_{2}^{-}\right]^{T}=\mathbf{A}^{-1}(\vec{b}-\vec{c})$ are the equilibrium points of $\vec{f}_{+}^{c}(\cdot)$ and $\vec{f}_{-}^{c}(\cdot)$, respectively. Since the vector field on each side of the switching line are transversal to $O$, the flows corresponding to these vector fields are diffeomorphisms, and the solutions to the systems of differential equations on each side of the switching line are continuous with respect to the initial condition, $P(\cdot)$ is a diffeomorphism [21].

Consider the flow diagram shown in Fig. 2, and let $y^{\prime \prime}$ denote the point in $S_{0_{1}}$ that is mapped to point $T 2$ through the Poincare map defined above. Note that $y^{\prime \prime} \in O, P\left(y^{\prime \prime}\right)=T 2$, and $T 2<y^{\prime \prime}$, thus $P\left(y^{\prime \prime}\right)-y^{\prime \prime}<0$. Similarly, let $y^{\prime}$ be the point in $O$ that is mapped to the $x_{2}$-intercept of $k 1$, through $P(\cdot)$. Then $P\left(y^{\prime}\right)-y^{\prime}>0$. Note that $y^{\prime \prime}$ and $y^{\prime}$ exist for the flow diagram shown in Fig. 2 since the flows corresponding to the vector fields on both sides of the switching line are diffeomorphisms. Now, since $P(y)$ is continuous in $y$, the intermediate value theorem requires that for some $y \in O, P(y)=y$. Note that trajectories of system (2) emanating from the $x_{2}$-axis above point $y^{\prime}$ will grow to infinity and that any limit cycle of system (2) must intersect the $x_{2}$-axis below $y^{\prime}$.

Now, let $y^{\star}$ be the point closest to $y^{\prime}$ such that $P\left(y^{\star}\right)=y^{\star}$, then the slope of $P(\cdot)$ at $y^{\star}$ is greater that one $e^{20}$. Thus, the limit cycle of system (2) corresponding to $P\left(y^{\star}\right)=y^{\star}$ is unstable. Furthermore, continuous time trajectories of system (2) outside this unstable limit cycle grow to infinity.

\footnotetext{
${ }^{20}$ This follows from the fact that $P(\cdot)$ is smooth and $P\left(y^{\prime}\right)>y^{\prime}$.
} 


\section{ACKNOWLEDGMENT}

The authors would like to thank Prof. B. Boser, Dr. M. Varghese, and Dr. S. Hein for valuable discussions and comments. We would also like to express our gratitude to the anonymous reviewers for their comments and suggestions.

\section{REFERENCES}

[1] R. M. Gray, "Spectral analysis of quantization noise in a single loop sigma delta modulator with de input," IEEE Trans. Commun., vol. 37 pp. 588-599, June 1989

[2] B. E. Boser and B. Wooley, "Quantization error spectrum of sigma delta modulators," in ISCAS, 1988 , pp. 2331-2334

[3] S. R. Norsworthy, "Effective dithering of sigma delta modulators," in ISCAS, San Diego, CA, May 1991, pp. 1304-1307.

[4] O. C. Feely, "An analytical study of the nonlinear dynamics of sigma delta analog to digital conversion," Ph.D. dissertation, Univ. Calif., Berkeley, 1992.

[5] M. Motamed, A. Zakhor, and S. Sanders, "Limit cycles and tone behavior of sigma delta modulators," 1992 DSP Workshop, Utica, IL, Sept. 1992.

[6] - "Tones, saturation, and SNR in double loop $\Sigma \Delta$ modulators," in ISCAS 1993 , pp. 1345-1348.

[7] R. Schreier, "On the use of chaos to reduce idle-channel tones in delta sigma modulators," IEEE Trans. Circuits Syst. I, vol. 41, pp. 539-547, Aug. 1994.

[8] S. Hein, "Exploiting chaos to suppress spurious tones in general doubleloop $\Sigma \Delta$ modulators," IEEE Trans. Circuits Syst. II, vol. 40, pp 651-659, Oct. 1993 .

[9] M. Motamed, S. Sanders, and A. Zakhor, "Analysis of tones in the double loop $\Sigma \Delta$ modulator with unstable filter dynamics," in ISCAS 1994, pp. 437-440.

[10] L. Risbo, "On the design of tone-free sigma delta modulators," IEEE Trans. Circuits Syst. II, vol. 42, pp. 52-55, Jan. 1995.

[11] C. Dunn and M. Sandler, "Linearising sigma-delta modulators using dither and chaos," in ISCAS 1995, pp. 625-628.

[12] S. Hein and A. Zakhor, "On the stability of sigma delta modulators," IEEE Trans. Signal Processing, pp. 2322-2348, July 1993.

[13] _ "Stability and scaling of double loop $\Sigma \Delta$ modulators," in ISCAS 1992 , pp. $1312-1315$.

[14] H. Wang, "A geometric view of $\Sigma \Delta$ modulations," IEEE Trans. Circuits Syst. II, vol. 39, pp. 402-405, June 1992.

[15] S. Pinault and P. Lopresti, "On the behavior of the double loop sigma delta modulator," IEEE Trans. Circuits Syst. II, vol. 40, pp. 467-479, Aug. 1993

[16] O. Feely, "Nonlinear dynamics of chaotic double-loop sigma-delta modulation," in ISCAS 1994, pp. 101-104.

[17] F. M. Callier and C. A. Desoer, Linear System Theory. New York: Springer-Verlag, 1991.

[18] C. Chen, Linear System Theory and Design. New York: HRW, 1984.

[19] A. F. Filippov, "Differential equations with discontinuous right-hand side," Amer. Math. Society Trans., vol. 42, Ser. 2, pp. 199-231, 1964.

[20] M. W. Hirsch and S. Smale, Differential Equations, Dynamical Systems, and Linear Algebra. New York: Academic, 1974.

[21] T. S. Parker and L. O. Chua, Practical Numerical Algorithms for Chaotic Systems. New York: Springer-Verlag, 1989.

[22] I. Z. Tsypkin, Relay Control Systems. Cambridge, MA: Cambridge Univ. Press, 1984.

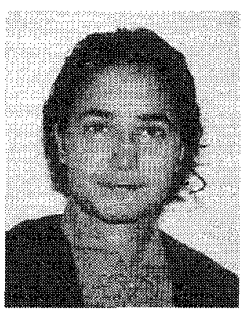

Mariam Motamed received the B.S. degree from the University of California, Santa Barbara and the S.M. degree from the Massachusetts Institute of Technology, Cambridge, in electrical engineering, in 1987 and 1989, respectively. She received the Ph.D. degree in electrical engineering from the University of California, Berkeley, in 1996.

From 1989 to 1990 she worked as an application engineer at at the chromatography division of Varian Associates, Walnut Creek, CA. She is currently with Philips Semiconductors, Sunnyvale, CA. Her research interests include design methodologies for oversampled data converters, digital signal processing and dynamical systems.

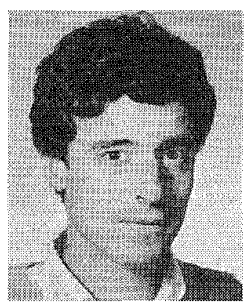

Seth Sanders received S.B. degrees in electrical engineering and physic $;$ in 1981, and the S.M. and Ph.D. degrees in electrical engineering in 1985 and 1989 , respectively, all from the Massachusetts Institute of Technology, Cambridge.

Between his S.B. and S.M. degrees, he worked as a design engineer at the Honeywell Test Instrument Division, Denver, $\mathrm{CO}$. $\mathrm{He}$ is presently Assistan Professor with the Department of Electrical Engineering and Computer Sciences, University of California, Berkeley. His research interests are in power electronics, variable speed drive systems, simulation, and in nonlinear circuit and system theory as related to the power: electronics field. During the 1992-1993 academic year, he was on industrial leave with National Semiconductor, Santa Clara, CA.

Dr. Sanders was a recipient of the NSF Young Investigator Award in 1993 and presently serves as Chair of the IEEE Technical Committee on Computers in Power Electronics.

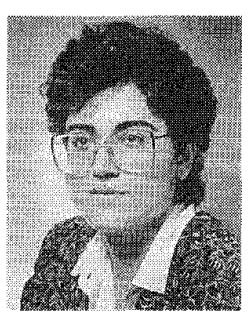

Avideh Zakhor received the B.S. degree from the California Institute of Technology, Pasadena, and the S.M. and Ph.D. degrees from the Massachusetts Institute of Technology, Cambridge, all in electrical engineering, in 1983, 1985, and 1987. respectively.

In 1988, she joined the Faculty at the University of California, Berkeley, where she is currently Associate Professor with the Department of Electrical Engineering and Computer Sciences. Her research interests are in the general area of signal processing and its applications to images and video, and biomedical data. She has been a consultant to a number of industrial organizations, holds four U.S. patents, and is the coauthor of the book, Oversampled $A / D$ Converters with Soren Hein.

Dr. Zakhor was a General Motors scholar from 1982 to 1983 . She received the Henry Ford Engineering Award and Caltech Prize in 1983, was a Hertz fellow from 1984 to 1988 , and received the Presidential Young Investigators (PYI) award, IBM Junior Faculty Development award, Analog Devices Junior Faculty Development award in 1990, and the Office of Naval Research (ONR) Young Investigator award in 1992 . She is currently a member of the technical committee for image and multidimensional digital signal processing. 